\title{
Síndrome hepatorrenal: fisiopatología, diagnóstico y manejo
}

\section{Pathophysiology, Diagnosis and Management of Hepatorenal Syndrome}

\author{
Juan Ricardo Ospina T., ${ }^{1}$ Juan Carlos Restrepo G., MD²
}

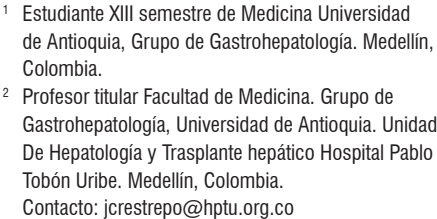
de Antioquia, Grupo de Gastrohepatología. Medellín, Colombia.

2 Profesor titular Facultad de Medicina. Grupo de Gastrohepatología, Universidad de Antioquia. Unidad De Hepatología y Trasplante hepático Hospital Pablo Tobón Uribe. Medellín, Colombia.

Contacto: jcrestrepo@hptu.org.co

\begin{abstract}
Resumen
El síndrome hepatorrenal es la forma de disfunción renal que complica a los pacientes con enfermedad hepática avanzada o insuficiencia hepática aguda. En él se presentan alteraciones notables en la circulación renal arterial, lo cual conlleva a una elevación progresiva de los niveles de creatinina y ascitis. En la actualidad su esquema de tratamiento farmacológico es limitado, por lo que la mejor opción terapéutica resulta ser el trasplante hepático.
\end{abstract}

\author{
Palabras claves \\ Síndrome hepatorrenal, cirrosis, diagnóstico, tratamiento.
}

\section{Abstract}

Hepatorenal syndrome is a form of renal dysfunction develops as a complication in patients with advanced liver disease and in patients with acute liver failure. Significant alterations in renal blood flow lead to progressively increasing levels of creatinine and to ascites. Currently, the best treatment option is liver transplantation because options for treatment with drugs are very limited.

Keywords

Hepatorenal syndrome, cirrhosis, diagnosis, treatment.

\section{INTRODUCCIÓN}

El hígado y el riñón son dos órganos que juegan un papel primordial en la hemostasis corporal. El primero es la piedra angular de las actividades metabólicas corporales y el segundo es el encargado de eliminar los productos de desecho generados en los diferentes ciclos metabólicos del organismo, así como de controlar el volumen y la composición de los líquidos en los diferentes compartimientos (1). Existen patologías en las cuales se presenta deterioro conjunto de la función de ambos órganos y una de estas es el síndrome hepatorrenal (SHR), el cual es definido como una insuficiencia renal funcional reversible en ausencia de daño del parénquima renal (1-4). Se sabe que el desarrollo del SHR eleva significativamente la mortalidad y la tasa de complicaciones posterior al trasplante hepático; hecho por el cual, desde el año 2002, se comenzó a implementar una escala para valorar la gravedad de la cirrosis y priorizar a los pacientes en la lista de trasplante hepático conocida como MELD (Model for Endstage Liver Disease) $(3,4)$.

El término SHR fue usado por primera vez en 1939 para describir la disfunción renal que seguía a la cirugía biliar y al trauma hepático. Después, entre 1994 y 1996, se realizó una reunión en Chicago donde se estandarizó la nomenclatura y se propusieron los criterios diagnósticos para la ascitis refractaria y el SHR. Para 2007 se llevó a cabo la 
quincuagésima sexta reunión de la asociación americana para el estudio de las enfermedades hepáticas. Allí se creó un grupo de estudio sobre el SHR, que reviso los criterios diagnósticos previamente establecidos (5).

\section{EPIDEMIOLOGÍA Y FACTORES DE RIESGO}

\section{Epidemiología}

El SHR se presenta en cerca del 10\% de los pacientes con cirrosis avanzada o insuficiencia hepática aguda (1-9). Ahora bien, se ha establecido que de los pacientes con cirrosis y ascitis un 18\% desarrollaran el síndrome a un año y $39 \%$ lo harán a cinco años (3), además, en este grupo de pacientes el riesgo aumenta cuando hay ascitis refractaria (10).

\section{Factores de riesgo}

- Peritonitis bacteriana espontánea (PBE): en esta afección, la antibioticoterapia profiláctica ha demostrado ser importante para la prevención del daño renal secundario a cirrosis. Estudios recientes han demostrado que la administración de norfloxacino como profilaxis reduce las probabilidades de SHR a un año (28\% grupo tratante frente al $41 \%$ grupo control) (6). En igual medida, el manejo con albúmina en estos pacientes ha mostrado ser un elemento preventivo. Estudios demostraron que los pacientes con PBE que reciben albúmina en dosis de $1,5 \mathrm{~g} / \mathrm{kg}$ al momento del diagnóstico de PBE y $1 \mathrm{~g} / \mathrm{kg}$ al tercer día, muestran una reducción de $66 \%$ en la incidencia de SHR $(5,7)$.

- Hepatitis alcohólica: esta entidad aumenta el riesgo del SHR, aunque hay evidencia de que esto puede ser reducido mediante el uso de medicamentos con efecto hematológico, y no de esteroides. Un estudio realizado en la India encontró que la administración de pentoxifilina $400 \mathrm{mg}$ tres veces al día en pacientes con hepatitis alcohólica aguda grave se asociaba con disminución en la incidencia de SHR (8).

- Insuficiencia hepática fulminante (9)

- Diuresis excesiva (9)

- Paracentesis de gran volumen en ausencia de expansores de volumen tipo albúmina (9)

- Hemorragia gastrointestinal (9)

- Administración de medios de contraste (10)

- Antibióticos nefrotóxicos (10)

- Antiinflamatorios no esteroideos (AINE) (10).

\section{FISIOPATOLOGÍA}

Para comprender el SHR hay que conocer la enfermedad cirrótica y los elementos primordiales en el desarrollo de la misma; es decir, los cambios propios de los pacientes con cirrosis, la ascitis y la hipertensión portal (11).

Cirrosis es el estadio final de todas las enfermedades hepáticas crónicas progresivas y se caracteriza por la distorsión en la arquitectura y la microcirculación hepática (12). Es una enfermedad con múltiples etiologías: metabólicas, toxicas, infecciosas, autoinmunes, farmacológicas, genéticas, biliares, vasculares o criptogénica. Sin embargo, sus tres principales causas son el abuso de alcohol, la enfermedad por hígado graso no alcohólico y la hepatitis viral crónica (12-14).

La patología cirrótica inicia con una fase asintomática en donde los pacientes, a pesar de no tener signos ni síntomas, pueden desarrollar hipertensión portal y varices esofágicas (12,14-16). Con el progreso de la enfermedad la presión portal aumenta, lo cual torna al paciente sintomático y facilita que aparezcan las complicaciones propias de este trastorno: ascitis, hemorragia por varices esofágicas, $\mathrm{SHR}$ y/o hepatopulmonar, encefalopatía hepática y PBE (12-18). Habiendo entrado en contexto en lo que respecta la cirrosis podemos centrarnos en los factores con rol importante mencionados anteriormente:

\section{Hipertensión portal y ascitis}

El aumento en la presión de la porta incrementa la producción y la liberación en la circulación arterial esplénica de vasodilatadores endógenos como el óxido nítrico (NO), monóxido de carbono (CO) y cannabinoides, lo cual a su vez incrementa el volumen plasmático local y termina disminuyendo rápido la presión arterial media (19). Para contrarrestar ello el cuerpo aumenta la fracción de eyección, libera vasopresina, activa el sistema nervioso simpático y los sistemas de vasoconstricción endógena como el sistema renina-angiotensina-aldosterona, con lo cual se genera contracción vascular a nivel renal, que permite disminuir la tasa de filtración glomerular (TFG) y retener tanto agua como sodio (20). Esto explica el desarrollo de ascitis, favorece la translocación bacteriana y lleva a PBE y SHR $(21,22)$. Es de resaltar que esta translocación y la endotoxemia resultante son responsables de estimular una respuesta proinflamatoria caracterizada por producción de citocinas como el factor de necrosis tumoral alfa e interleucina-6, lo que facilita la producción de óxido nítrico, que termina empeorando la vasodilatación del lecho esplácnico $(3,4)$.

\section{Cambios estructurales y funcionales del paciente cirrótico}

Entre estos se encuentran la hipertrofia cardíaca, disfunción diastólica y la menor respuesta a estímulos ionotrópico y cronotrópico; todos ellos asociados a una menor fracción de eyección y, por ende, a falla renal (23-25). 
La activación de los sistemas vasoconstrictores, ante el estímulo vasodilatador, representa el mediador principal de disfunción tubular y hemodinámica del riñón (26). Este potente efecto lleva a isquemia renal, lo cual estimula la liberación de endotelina- 1 y con ella la hiperactividad del sistema nervioso simpático. Todo ello genera un ciclo que termina agravando la TFG $(27,28)$. Ahora bien, la disminución en la TFG es un evento tardío, puesto que los sucesos previos al desarrollo del SHR son la hiponatremia y ascitis, circunstancias que han sido consideradas como marcadores pronósticos independientes de la creatinina (Figura 1) (29-32).

\section{DIAGNÓSTICO}

Hasta el 2015, el diagnóstico del SHR se establecía cuando un paciente cumplía los seis criterios que habían sido revisados en 2007(3,33):

1. Cirrosis + ascitis

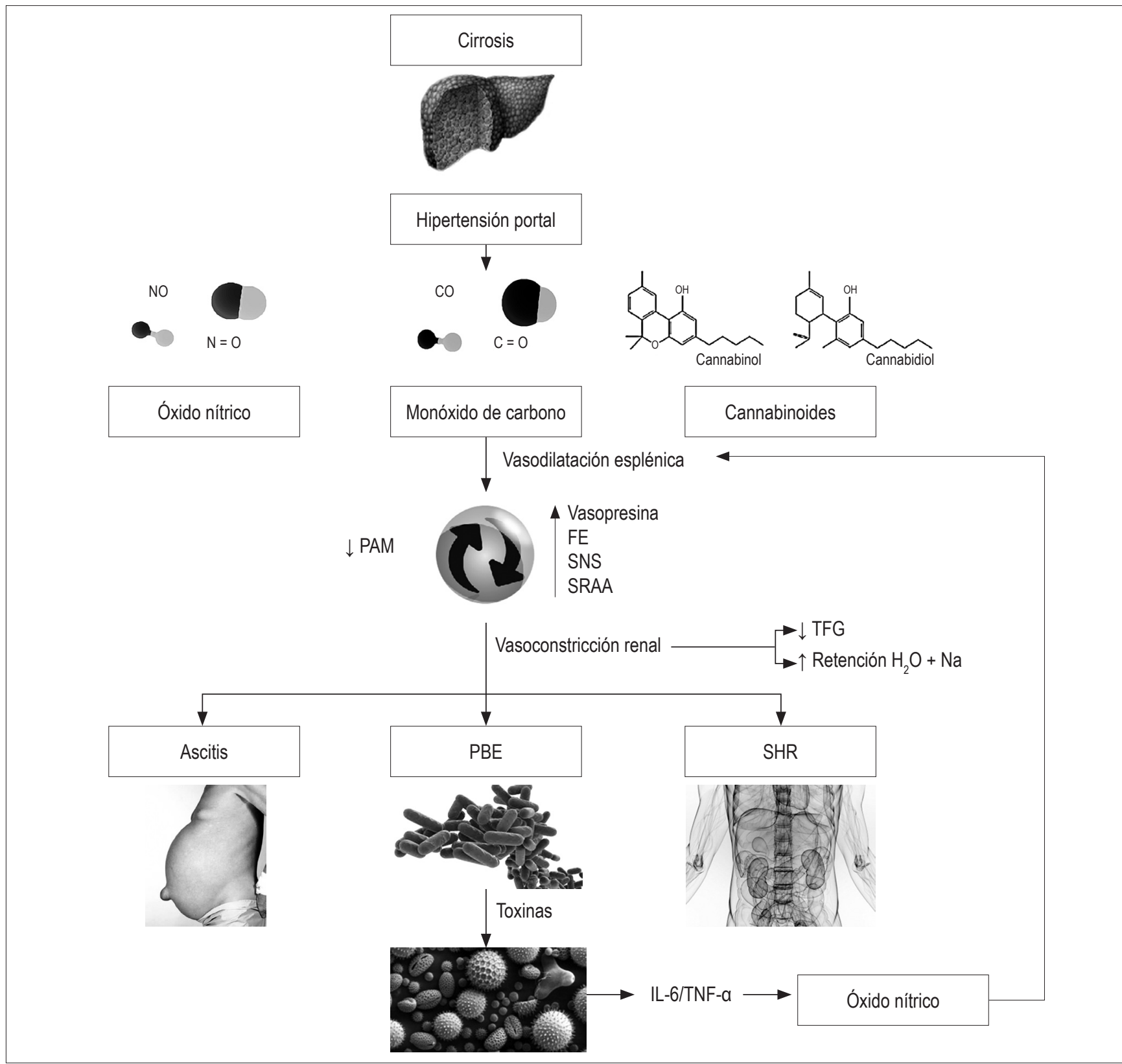

Figura 1. Esquema del proceso fisiopatológico del síndrome hepatorrenal. FE: fracción de eyección; IL-6: interleucina 6; PAM: presión arterial media; PBE: peritonitis bacteriana espontanea; SHR: síndrome hepatorrenal; SNS: sistema nervioso simpático; SRAA: sistema renina angiotensina aldosterona; TNF- $\alpha$ : factor de necrosis tumoral alfa. 
2. Creatinina sérica $>1,5 \mathrm{mg} / \mathrm{dL}$

3. Ausencia de mejoría en las cifras de creatinina sérica $(<1,5 \mathrm{mg} / \mathrm{dL})$ después de al menos 2 días sin diuréticos y expansión de volumen con albúmina (dosis $1 \mathrm{~g} / \mathrm{kg} /$ día, dosis máxima $100 \mathrm{~g} /$ día)

4. Ausencia de choque

5. Ausencia de tratamiento actual o reciente con nefrotóxicos

6. Ausencia de enfermedad parenquimatosa renal (proteinuria $<500 \mathrm{mg} /$ día, microhematuria $<50$ eritrocitos/ campo de alta resolución, ultrasonido renal normal)

Una vez hecho el diagnóstico se debe definir a cuál de los subtipos de SHR pertenece el paciente $(3,7,33,35)$ :

- Tipo 1: caracterizado por ser rápidamente progresivo, duplicar la creatinina sérica inicial a un valor superior a $2,5 \mathrm{mg} / \mathrm{dL}$ en un período menor a 2 semanas, generar disfunción circulatoria profunda, llevar a hiponatremia dilucional, tener mortalidad elevada con una tasa de supervivencia de tan solo $25 \%$ a un mes y $10 \%$ a tres meses si el paciente no recibe tratamiento, y ser precipitado por hepatitis alcohólica severa, sangrado gastrointestinal, sepsis o por infecciones como PBE.

- Tipo 2: caracterizado por ser lentamente progresivo, incrementar la creatinina entre $1,5-2,5 \mathrm{mg} / \mathrm{dL}$, tener una supervivencia de 6 a 12 meses y estar frecuentemente asociado con ascitis refractaria.

Sin embargo, en el presente año, el club internacional de ascitis (ICA) publicó los nuevos criterios para hacer el diagnóstico de insuficiencia renal aguda (IRA) en pacientes cirróticos. En estos, se modificó el umbral estándar de la creatinina sérica de $1,5 \mathrm{mg} / \mathrm{dL}$, que estaba siendo utilizado en los últimos años, con base en dos aspectos: $i$ ) que para tener un valor de creatinina sérica de $1,5 \mathrm{mg} / \mathrm{dL}$, la TFG debe estar marcadamente disminuida; ii) que al tener un umbral fijo, no se tienen en cuenta los cambios dinámicos en los niveles séricos de creatinina que ocurren días o semanas previas a lograr este umbral, aspectos que son necesarios para distinguir entre la lesión renal aguda y crónica (34).

Teniendo en cuenta estos aspectos, se establecieron los criterios diagnósticos de IRA en pacientes cirróticos. Además se fijaron tres estadios de la enfermedad, la progresión, la respuesta al tratamiento y se dieron recomendaciones para el manejo farmacológico precoz de esta patología (34):

- Diagnóstico: incremento de la creatinina sérica $\geq 0,3$ $\mathrm{mg} / \mathrm{dL}$ en un período de 48 horas o aumento en más de $50 \%$ de la creatinina sérica inicial (conocida o presumida) en un término de 7 días.

- Estadios:
- Estadio 1: incremento de la creatinina sérica mayor o igual a $0,3 \mathrm{mg} / \mathrm{dL}$ o mayor de 1,5 a 2 veces la basal.

- Estadio 2: incremento de la creatinina sérica mayor de 2 a 3 veces la basal.

- Estadio 3: incremento de la creatinina sérica mayor de 3 veces la basal o nivel de creatinina sérica mayor a $4 \mathrm{mg} / \mathrm{dL}$ con un incremento agudo mayor o igual a $0,3 \mathrm{mg} / \mathrm{dL}$ o inicio de terapia de reemplazo renal.

- Progresión-Regresión: la progresión es definida cuando el paciente aumenta su estadio y/o necesita terapia de reemplazo renal. La regresión se presenta cuando el paciente desciende de estadio.

- Respuesta al tratamiento:

- Sin respuesta: cuando no hay regresión de la falla renal aguda.

- Respuesta parcial: cuando hay regresión en el estadio con una reducción de la creatinina sérica $\geq 0,3$ $\mathrm{mg} / \mathrm{dL}$ por encima del valor basal.

- Respuesta total: cuando la creatinina retorna a un valor dentro de $0,3 \mathrm{mg} / \mathrm{dL}$ del basal.

- Se determinó que la creatinina sérica basal a usar puede ser cualquier medición que tenga el paciente dentro de los últimos 3 meses (si tiene varias, se usará la más cercana al ingreso hospitalario). Ahora, si el paciente no cuenta con mediciones se tomará como basal la que se mida al ingreso hospitalario.

- Así, de los criterios mencionados para el diagnóstico del SHR se reemplazó el valor fijo de creatinina sérica $>1,5 \mathrm{mg} / \mathrm{dL}$ por tener un diagnóstico de IRA, de acuerdo a los criterios establecidos por el ICA. Por otro lado, el ICA sugiere que solo se continúe usando el umbral de creatinina sérica de $1,5 \mathrm{mg} / \mathrm{dL}$ para establecer el pronóstico de los pacientes cirróticos con IRA que sean clasificados como estadio 1 , los cuales se deben ubicar en uno de dos grupos (34):

- Aquellos pacientes que al ser hospitalizados tienen creatinina sérica menor a $1,5 \mathrm{mg} / \mathrm{dL}$. La mortalidad a corto plazo en estos es similar a la de los pacientes sin IRA; además, tendrán más posibilidades de regresión de su trastorno renal.

- Aquellos pacientes con niveles de creatinina sérica superiores a $1,5 \mathrm{mg} / \mathrm{dL}$. La mortalidad a corto plazo en estos es mayor a la de los pacientes sin IRA.

\section{TRATAMIENTO}

A pesar de los avances en el conocimiento del SHR, el abanico de posibilidades terapéuticas sigue siendo muy limitado; por el momento, estas se basan en recomendaciones 
generales, tratamientos farmacológicos, derivación portosistémica intrahepática transyugular (TIPS), trasplante hepático y seguir las indicaciones propuestas por el ICA.

\section{Recomendaciones generales (35)}

- Restringir el consumo de sal a $80-120 \mathrm{mmol} /$ día

- No restringir el consumo de líquidos

- Eliminar nefrotóxicos identificados. Si el paciente está usando diuréticos se debe recordar que entre sus efectos adversos esta la insuficiencia renal, encefalopatía e hiponatremia. Si esta última se presenta siempre se deben suspender si el sodio sérico es menor de $120 \mathrm{mEq} / \mathrm{L}$.

- Ante ascitis grado 1 o 2 se pueden utilizar dosis crecientes de diuréticos para controlarla (36) o adicionar clonidina para disminuir el requerimiento diurético y disminuir la re acumulación del líquido ascítico (37).

- Ante ascitis grado 3 o ascitis refractaria (ausencia de mejoría de la ascitis usando dosis elevadas de diuréticos), la primera línea de manejo será realizar paracentesis de gran volumen $(\mathrm{X}>5$ litros $)$. El problema de este procedimiento será el riesgo de desarrollar SHR, por lo que se debe hacer prevención usando la terapia con infusión de albúmina reponiendo $8 \mathrm{~g} / \mathrm{L}$ extraído. Con esta se busca aumentar la presión de perfusión renal, mejorar los niveles de creatinina y facilitar la excreción fraccional de sodio (38).

\section{Recomendaciones propuestas por el ICA para el control de la IRA en el cirrótico}

\section{Paciente en estadio 1 (34)}

- Evaluar que medicamentos consume el paciente y retirar las drogas nefrotóxicas como AINE, IECA, ARA2 y vasodilatadores. Los diuréticos se pueden reducir o retirar según las condiciones del paciente.

- Ante hipovolemia se debe expandir el volumen plasmático con cristaloides, albúmina o sangre. Esta última se usa ante IRA secundaria a sangrado gastrointestinal.

- Controlar las infecciones bacterianas en el paciente. Si hay signos de PBE se debe iniciar manejo antibiótico e idealmente asociarlos a infusión de albúmina $(37,38)$.

- De estos pacientes, aquellos que presenten respuesta total al tratamiento deben ser seguidos midiendo niveles de creatinina sérica cada 2 a 4 días durante la hospitalización, y luego del alta cada 2 a 4 semanas durante los primeros 6 meses para identificar nuevos episodios IRA. Ahora bien, en aquellos pacientes que no progresan ni tienen respuesta total al tratamiento no hay consenso en el manejo, por lo que hacen falta estudios.

\section{Paciente en estadio 2-3 o estadio 1 de}

\section{IRA que no presento respuesta (34)}

- Se deben retirar los diuréticos en caso de que no se hubieran retirado, y expandir el volumen plasmático con albúmina intravenosa a dosis de $1 \mathrm{~g} / \mathrm{kg} / 2$ días consecutivos (dosis máxima $100 \mathrm{~g} /$ día) para tratar la IRA prerrenal.

- Se debe hacer el diagnóstico diferencial observando especialmente si el paciente cumple los criterios diagnósticos del SHR. Si no los cumple, se debe buscar la causa de IRA; si los cumple, se debe iniciar albúmina y vasoconstrictores (idealmente terlipresina) independiente del valor de creatinina sérica.

\section{Trasplante hepático}

Se debe considerar este tratamiento en todo paciente cirrótico con ascitis (especialmente en caso de ascitis refractaria: $50 \%$ de estos muere en 6 meses y el $75 \%$ al año), PBE y/o SHR cuando no hay consumo actual de alcohol o enfermedad maligna asociada. El trasplante ha demostrado aumentar la supervivencia de los pacientes y en la actualidad se cuenta con el MELD para priorizar a los pacientes en la lista de trasplante (3-5,11). Sobre el SHR se debe tener en cuenta que el trasplante es el tratamiento de elección ya que revierte la patología. Ahora bien, diferentes estudios han demostrado que los pacientes trasplantados que presentaban SHR previo al procedimiento, tenían morbilidad superior luego de la cirugía respecto a los pacientes trasplantados que no cursaban con SHR. Del mismo modo, mostraban que en un período de 5 años luego del trasplante la mortalidad era del $32 \%$ en los pacientes con enfermedad hepática avanzada y disfunción pre renal $(5,11)$. En cuanto al trasplante renal simultáneo, la recomendación actual es realizarlo a todos los pacientes cirróticos con clasificación AKI 3 o enfermedad renal crónica con TFG menor de 35 $\mathrm{mL} / \mathrm{min}$ por más de 4 semanas o IRA con requerimiento de diálisis por más de 8 semanas (11-38).

\section{Terlipresina}

Es un análogo de la vasopresina que induce vasoconstricción. Se cree que mejora la perfusión renal en el SHR porque reduce la vasodilatación esplénica; por ende, la disminución del flujo renal (39). Hay varios ensayos clínicos controlados y aleatorizados que han demostrado que revierte el SHR tipo 1 , lo cual genera una leve reducción en la mortalidad a corto plazo (39). A diferencia de ello, en el SHR tipo 2, la falla renal retorna una vez se suspende el medicamento, por lo que se ha propuesto su uso en estos pacientes solo como puente de tratamiento previo al trasplante hepático (36). Los últimos 
estudios han mostrado que se puede emplear conjuntamente con la albúmina en pacientes con SHR tipo 1 y sepsis asociada, ya que ello incrementa el volumen arterial sanguíneo y con ello la precarga (40).

Tal y como lo recomendó el ICA, se debe iniciar este medicamento tan pronto como se diagnostique el SHR, con una dosis inicial de $1 \mathrm{mg} / 4-6$ horas. Si la creatinina sérica no disminuye más del $25 \%$ de su valor, luego de 2 días de tratamiento, se debe duplicar la dosis cada 48 horas hasta un máximo de $12 \mathrm{mg}$ /día. Se debe detener su uso si la creatinina sérica no disminuye al menos $50 \%$, luego de 7 días de usar el fármaco a dosis máxima, o si no se produce el descenso después de los 3 primeros días. En los pacientes con respuesta inmediata, el tratamiento debe continuarse hasta la reversión del síndrome o hasta un efecto máximo de 14 días (2). Hasta un 30\% de los pacientes presentaran efectos adversos a este medicamento, pero solo en el $4 \%$ se deberá detener el tratamiento debido a infarto agudo de miocardio o arritmias malignas, ambas contraindicaciones absolutas (11).

\section{Norepinefrina}

Es una catecolamina que actúa sobre los receptores adrenérgicos y ha demostrado ser tan efectiva como la terlipresina en el control del SHR tipo 1. Un estudio piloto usó el medicamento en infusión en dosis de 0,5-3 mg/hora, asociada a expansión con albúmina. Encontraron resultados similares a los obtenidos con Terlipresina (2). Sin embargo, este medicamento tiene más desventajas, razón por la cual su utilización se reserva para el momento en que no se tenga disponible la terlipresina $(11,35)$.

\section{Midodrina y octreotida}

La midodrina es un profármaco que actúa como agonista selectivo de los receptores $\alpha$-1 periféricos generando vasoconstricción y aumentando con ello la presión arterial. Mientras que la octreotida es un análogo de la somatostatina, que actúa como inhibidor de la secreción de péptidos sintetizados por el sistema endocrino gastroenteropancreático, con lo cual logra disminuir el flujo sanguíneo esplénico. Dos estudios piloto han evaluado el tratamiento con midodrina + octreotida y albúmina en infusión para el manejo del SHR tipo 1. Los resultados mostraron que estos medicamentos generan un efecto similar a la terlipresina, aunque la respuesta fue más lenta. A partir de esto se ha propuesto usarlo cuando no se tiene disponible la terlipresina para manejar el SHR tipo 1, aunque no hay un consenso en cuanto a su dosis (11). Frente al SHR tipo 2, el tra- tamiento con vasoconstrictores ha sido menos estudiado y solo se sabe que en los estudios realizados la mayoría de los pacientes que usaron la terlipresina consiguieron normalizar la creatinina sérica, pero al retirar el fármaco reapareció el fracaso renal, por lo que se requieren más estudios (11).

\section{TIPS}

Este es un procedimiento que consiste en crear una comunicación entre las circulaciones portal y sistémica mediante una prótesis autoexpandible entre una vena suprahepática y una rama portal. Con esto se consigue disminuir la presión portal y los eventos que inducen la ascitis (11). Este procedimiento está avalado, así como la paracentesis evacuante, para los pacientes con SHR tipo 2 que desarrollan ascitis refractaria al tratamiento con diuréticos. Los estudios han demostrado que su uso disminuye la tasa de recurrencia de la ascitis y mejora la sobrevida comparada con la paracentesis evacuante. El problema de hacerlo es que puede causar falla cardíaca y encefalopatía hepática, por lo que su uso no está libre de complicaciones (11). En el momento está contraindicado en pacientes con encefalopatía crónica, ChildPugh superior a 12, edad mayor de 70 años o disfunción cardíaca con fracción de eyección menor al 50\% (11).

\section{CONCLUSIÓN}

La cirrosis día a día se afianza como una de las patologías más prevalentes en el mundo y sus múltiples complicaciones cobran la vida de un mayor número de personas anualmente. Una de estas complicaciones es el SHR, el cual se observa en el $10 \%$ de estos pacientes y representa un indicador de mal pronóstico. En el presente año, la asociación internacional de ascitis publicó los nuevos criterios para hacer el diagnóstico de falla renal aguda en cirróticos, con lo cual se espera tener una guía para orientar el manejo de estos pacientes e impactar la morbilidad y mortalidad que caracteriza a esta entidad. A pesar de los múltiples esfuerzos investigativos en el tema todavía el abanico terapéutico es muy reducido, por lo que hacen falta nuevos estudios que arrojen otras opciones terapéuticas para ofrecerles a estos pacientes.

\section{Agradecimientos}

Ofrecemos agradecimientos al grupo de investigación de gastrohepatología y al doctor Juan Carlos Restrepo Gutiérrez, a quien especialmente dirigimos un inmenso reconocimiento por su labor académica, calidad humana y enseñanza clínica. Los autores también agradecen al Proyecto de Sostenibilidad, Vicerrectoría de Investigación, Universidad de Antioquia. 


\section{REFERENCIAS}

1. Longo DL, Fauci AS, Kasper DL, Hauster SL, Jamenson JL, Harrison JL. Principios de Medicina Interna. 18.a edición. México, D.F. McGRAW-HILL. 2012. p. 2601.

2. Toranzo Labrada R, González Castilla R, García Medina J. Síndrome hepatorrenal: diagnóstico y tratamiento. Medisan. 2012;16(5):787.

3. Higuera-de la Tijera MF et al. Conceptos actuales en síndrome Hepatorrenal. Rev Med Hosp Gen Méx. 2011;74(1):42-9.

4. Ginès $\mathrm{P}$, Schrier RW. Renal failure in cirrhosis. N Engl J Med. 2009;361(13):1279-90.

5. Salerno F, Gerbes A, Ginès P, Wong F, Arroyo V. Diagnosis, prevention and treatment of hepatorenal syndrome in cirrhosis. Gut. septiembre de 2007;56(9):1310-8.

6. Muñoz SJ. The hepatorenal syndrome. Med Clin N Am 2008;92:813-837

7. Ginés P, Cárdenas A, Arroyo V, Rodés J. Management of cirrhosis and ascites. N Engl J Med 2004;350:1646-54.

8. De BK, Gangopadhyay S, Dutta D, et al. Pentoxifylline versus prednisolone for severe alcoholic hepatitis: A randomized controlled trial. World J Gastroenterol. 2009; 15:1613-9.

9. Craig E. Gordon. Hepatorenal Syndrome. HospMedClin. 2012;1:e62-e68.

10. Fowler C. Management of patients with complications of cirrhosis. Nurse Pract. 2013;38(4):14-21-23.

11. Egerod Israelsen M, Gluud LL, Krag A. Acute kidney injury and hepatorenal syndrome in cirrhosis. J Gastroenterol Hepatol. febrero de 2015;30(2):236-43.

12. García Bueya, F. González Mateosb y R. Moreno-Oteroa. Cirrosis hepática. Medicine. 2012;11(11):625-33.

13. Lefton HB, Rosa A, Cohen M. Diagnosis and epidemiology of cirrhosis. Med Clin North Am. 2009;93(4):787-799, vii.

14. de Franchis R, Baveno V Faculty. Revising consensus in portal hypertension: report of the Baveno $\mathrm{V}$ consensus workshop on methodology of diagnosis and therapy in portal hypertension. J Hepatol. 2010;53(4):762-8.

15. Benvegnù L, Gios M, Boccato $S$, Alberti A. Natural history of compensated viral cirrhosis: a prospective study on the incidence and hierarchy of major complications. Gut. 2004;53:744-9.

16. D’Amico G, García-Tsao G, Pagliaro L. Natural history and prognostic indicators of survival in cirrhosis: a systematic review of 118 studies. J Hepatol. 2006;44:217-31.

17. Nagula S, Jain D, Groszmann RJ, García-Tsao G. Histologicalhemodynamic correlation in cirrhosis-a histological classification of the severity of cirrhosis. J Hepatol. 2006;44:111-7.

18. De Franchis R, Baveno V Faculty. Revising consensus in portal hypertension: report of the Baveno $\mathrm{V}$ consensus workshop on methodology of diagnosis and therapy in portal hypertension. J Hepatol. 2010;53:762-8.

19. Brinch K, Moller S, Bendtsen F, Becker U, Henriksen JH. Plasma volume expansion by albumin in cirrhosis. Relation to blood volume distribution, arterial compliance and severity of disease. J. Hepatol. 2003;39:24-31.
20. Gines P, Schrier RW. Renal failure in cirrhosis. N. Engl. J. Med. 2009;361:1279-90.

21. Wiest R, Lawson M, Geuking M. Pathological bacterial translocation in liver cirrhosis. J. Hepatol. 2013; 60:197-209.

22. Madsen BS, Havelund T, Krag A. Targeting the gut-liver axis in cirrhosis: antibiotics and non-selective $\beta$-blockers. Adv Ther. 2013;30(7):659-70.

23. Ruiz-del-Arbol L, Urman J, Fernández J, González M, Navasa M, Monescillo A, et al. Systemic, renal, and hepatic hemodynamic derangement in cirrhotic patients with spontaneous bacterial peritonitis. Hepatology. 2003;38(5):1210-8.

24. Ruiz-del-Arbol L, Urman J, Fernández J, González M, Navasa M, Monescillo A, et al. Systemic, renal, and hepatic hemodynamic derangement in cirrhotic patients with spontaneous bacterial peritonitis. Hepatology. 2003;38(5):1210-8.

25. Mackelaite L, Alsauskas ZC, Ranganna K. Renal failure in patients with cirrhosis. Med Clin North Am. 2009;93(4):855869, viii.

26. Guevara M, Bru C, Ginès P, Fernández-Esparrach G, Sort P, Bataller R, et al. Increased cerebrovascular resistance in cirrhotic patients with ascites. Hepatology. 1998;28(1):39-44.

27. Arroyo V, Colmenero J. Ascites and hepatorenal syndrome in cirrhosis: pathophysiological basis of therapy and current management. J Hepatol. 2003;38 Suppl 1:S69-89.

28. Faber JE, Brody MJ. Afferent renal nerve-dependent hypertension following acute renal artery stenosis in the conscious rat. Circ Res. 1985;57(5):676-88.

29. Kim WR, Biggins SW, Kremers WK, Wiesner RH, Kamath PS, Benson JT, et al. Hyponatremia and mortality among patients on the liver-transplant waiting list. N Engl J Med. 2008;359(10):1018-26.

30. Heuman DM, Abou-Assi SG, Habib A, Williams LM, Stravitz RT, Sanyal AJ, et al. Persistent ascites and low serum sodium identify patients with cirrhosis and low MELD scores who are at high risk for early death. Hepatology. 2004;40(4):802-10.

31. Hasper D, Jörres A. New insights into the management of hepato-renal syndrome. Liver Int. 2011;31 Suppl 3:27-30.

32. Arroyo V, Colmenero J. Ascites and hepatorenal syndrome in cirrhosis: pathophysiological basis of therapy and current management. J Hepatol. 2003;38 Suppl 1:S69-89.

33. Pérez RM. Síndrome hepatorrenal: Enfoque actual. GastrLatinoam. 2006; 17:211-217.

34. Angeli $P$, Ginès P, Wong F, Bernardi M, Boyer TD, Gerbes A, et al. Diagnosis and management of acute kidney injury in patients with cirrhosis: revised consensus recommendations of the International Club of Ascites. J Hepatol. 2015;62(4):968-74.

35. Salerno F, Gerbes A, Ginès P, Wong F, Arroyo V. Diagnosis, prevention and treatment of hepatorenal syndrome in cirrhosis. Gut. 2007;56(9):1310-8.

36. European Association for the Study of the Liver. EASL clinical practice guidelines on the management of ascites, spontaneous bacterial peritonitis, and hepatorenal syndrome in cirrhosis. J Hepatol. 2010;53(3):397-417. 
37. Lenaerts A, Codden T, Meunier J-C, Henry J-P, Ligny G. Effects of clonidine on diuretic response in ascitic patients with cirrhosis and activation of sympathetic nervous system. Hepatology. 2006;44(4):844-9.

38. Leithead JA, Hayes PC, Ferguson JW. Review article: advances in the management of patients with cirrhosis and portal hypertension-related renal dysfunction. Aliment Pharmacol Ther. 2014;39(7):699-711.
39. Francoz C, Durand F. Type-1 hepatorenal syndrome in patients with cirrhosis and infection vs. sepsis-induced acute kidney injury: what matters? J Hepatol. mayo de 2014;60(5):907-9.

40. Rodríguez E, Elia C, Solà E, Barreto R, Graupera I, Andrealli A, et al. Terlipressin and albumin for type-1 hepatorenal syndrome associated with sepsis. J Hepatol. 2014;60(5):955-61. 